\title{
Migration of rigid spheres in a two-dimensional unidirectional shear flow of a second-order fluid
}

\author{
By B. P. HO AND L. G. LEAL \\ Chemical Engineering, California Institute of Technology, Pasadena
}

(Received 11 September 1974 and in revised form 10 May 1976)

The lateral migration of a neutrally buoyant rigid sphere suspended in a secondorder fluid is studied theoretically for unidirectional two-dimensional flows. The results demonstrate the existence of migration induced by normal stresses whenever there is a lateral variation of the shear rate in the undisturbed flow. The migration occurs in the direction of decreasing absolute shear rate, which is towards the centre-line for a plane Poiseuille flow and towards the outer cylinder wall for Couette flow. The direction of migration agrees with existing experimental data for a viscoelastic suspending fluid, and qualitative agreement is found between the theoretically predicted and experimentally measured sphere trajectories.

\section{Introduction}

Experimental and theoretical studies of suspensions have revealed two distinct mechanisms by which suspended particles can produce flow-rate dependent macroscopic behaviour. First, when the concentration of particles is spatially uniform, nonlinear macroscopic behaviour can result if the individual particles deform or preferentially orient in a manner which depends on the magnitude of the bulk velocity field. Macroscopic effects of this first type represent an intrinsic property of the suspension, and are thus reflected in the characteristic nonNewtonian form of the particle contribution to the bulk stress. Even when the particle contributions to the bulk stress are linear, however, the measured macroscopic variables in a particular viscometric experiment may still exhibit a nonlinear (flow-rate dependent) relationship. An example is the experimental measurements of flow rate $v s$. pressure drop for a dilute suspension of rigid spheres in a capillary viscometer by Segré \& Silberberg (1963). One cause for such behaviour is the presence, under appropriate conditions, of flow-induced lateral motion of the suspended particles. This motion tends to produce a non-uniform concentration distribution that depends both on the macroscopic flow rate and on the detailed geometry of the viscometer. In a recent theoretical investigation (Ho \& Leal 1974), we have considered the problem of inertia-induced migration of rigid spherical particles in a two-dimensional, quadratic, unidirectional shear flow (simple shear and plane Poiseuille flow are two examples) of a Newtonian fluid. The present communication is concerned with migration induced in similar circumstances as a result of non-Newtonian properties in the ambient suspending fluid. 
The phenomenon of lateral migration in non-Newtonian liquids has been studied experimentally by Mason and co-workers (Karnis \& Mason 1966; Gauthier, Goldsmith \& Mason $1971 a, b)$ and by Highgate \& Whorlow (1968, 1969). Mason's studies have demonstrated that the magnitude and even the direction of migration depend critically on the detailed rheological characteristies of the suspending fluid. Two distinct sets of results were obtained, one for purely viscous fluids (labelled pseudoplastic by Mason), which show a strong shear thinning of viscosity, but only very weak normal-stress or relaxation (recoil) phenomena, and the other for fully viscoelastic fluids (labelled elasticoviscous by Mason). In the latter case, which was studied by Karnis \& Mason (1966) for Poiseuille and Couette flows, rigid spherical particles were found to migrate in the direction of minimum shear rate, i.e. towards the centre-line in Poiseuille flow and towards the outer cylinder wall in Couette flow. On the other hand, when the suspending fluid is purely viscous, Gauthier et al. $(1971 a, b)$ have shown that migration of rigid spheres occurs in the opposite sense, i.e. towards the wall in Poiseuille flow and towards the inner cylinder in Couette flow. Detailed particle trajectories were measured in each case. Unfortunately, however, the available rheological data are both incomplete and of questionable accuracy (cf. Bartram 1973), so that the main value of these studies is qualitative. Related migration phenomena in viscoelastic fluids were also reported by Highgate \& Whorlow $(1968,1969)$, who studied Couette flow and the viscometrically interesting coneand-plate geometry. In the latter case, relatively rapid radial migration was observed which completely depleted the gap of the viscometer of suspended spheres after several minutes. The case of Couette flow was found in the experiment of Highgate \& Whorlow $(1968,1969)$ to exhibit an axially directed migration (i.e. at right angles to the cross-gap migration of Mason et al.) which again eventually depleted the gap of particles (although on a relatively longer time scale compared with the cone-and-plate case).

To date, no proper theoretical analysis has been reported for any of these phenomena, though it was suggested both by Highgate \& Whorlow $(1968,1969)$ and by Karnis \& Mason (1966) that a qualitatively relevant explanation could be obtained in the viscoelastic case by simply taking account of the net force produced on a sphere by the gradients of normal stress in the undisturbed flow. However, in the unidirectional flows under consideration, any gradients in the deviatoric normal stress components must be balanced by gradients in the pressure so that the net lateral force on any fluid element (and hence on the sphere) in the undisturbed flow is precisely zero. In addition, such an 'explanation' takes no account of the disturbance flow (and associated normal stresses) induced by the particle, which is of at least comparable magnitude to the undisturbed motions in its vicinity.

We consider here a complete theoretical analysis for plane quadratic shear flow of a second-order fluid. The analysis predicts that lateral migration will occur in the same direction as observed by Karnis \& Mason (1966) for fully viscoelastic fluids whenever there is a lateral variation of the shear rate of the undisturbed velocity profile. Since the non-Newtonian behaviour of a secondorder fluid in steady shear flow is limited to non-zero normal-stress differences, 
the present theory does partially confirm the theoretical ideas of Karnis \& Mason (1966), at least in the limited sense that lateral gradients of normal stress in the full velocity field are apparently responsible for the migration observed in strongly viscoelastic fluids. It is, of course, well known that the second-order fluid model is only relevant for very slow and thus nearly Newtonian flows. In particular, deviations of the normal stress components from their Newtonian values are strictly of only infinitesimal magnitude. In spite of this, we feel that the present analysis is of fundamental interest and at least qualitatively applicable to problems of practical interest. We have several reasons for this optimism. The first is the fact that the $n$ th-order fluid expansion, of which the second-order fluid includes the first two terms, is the common slow-flow limit of most other models of viscoelastic fluid behaviour. Thus, in spite of uncertainties which persist in the formulation of general viscoelastic fluid models, the $n$ th-order fluid 'models' are almost certainly relevant for flows which have a characteristic time scale which exceeds the intrinsic relaxation time of the material. Second, since the shear dependence of the viscosity does not enter the $n$ th-order fluid expansion until third order, the second-order model provides a rational basis for separating the effects of particle migration induced by normal stresses from that induced in the purely viscous case by gradients of the shear viscosity. In spite of the fact that the migration induced by normal stresses is necessarily restricted in magnitude by the nature of the second-order fluid model, there is no contribution at all from the dominant first-order (Newtonian) terms. Hence, under appropriate circumstances the small instantaneous effect can produce a major accumulative change in the particle motion. Finally, we call attention to the recent calculation by one of us (Leal 1975) of orbit drift for long slender particles in simple shear flow of a second-order fluid. This work has provided one example, which is closely related to the present work, of a case where the second-order fluid model gives quantitative agreement with experimental data, even for moderate to large shear rates, provided one uses measured values of the model parameters at the relevant bulk shear rate rather than the zero-shear values for which the model is strictly relevant. We suggest, therefore, that the qualitative physics of the normal-stress effects may not be too badly represented by the second-order fluid approximation over a reasonably wide range of shear rates. Comparison of the theoretical results from the present study with the experimental measurements of Karnis \& Mason (1966) supports this contention.

The detailed analysis required for the present problem follows rather closely that reported in our earlier study (Ho \& Leal 1974) of inertia-induced migration in a Newtonian fluid. Thus we concentrate our discussion primarily on those features which are unique to the non-Newtonian problem, and refer the reader to our earlier paper for other details of the calculation.

\section{The basic equations}

We consider a neutrally buoyant rigid sphere of radius $a$ freely suspended in an incompressible second-order fluid which is confined between two parallel plane walls separated by a distance $d$. The suspending fluid is assumed to be 
undergoing a steady unidirectional two-dimensional bulk flow which we denote by $\mathbf{V}$. The fluid density will be denoted by $\rho_{0}$, and the zero-shear viscosity by $\mu_{0}$. All quantities will be non-dimensionalized by the characteristic length $a$ and a characteristic velocity $G a$, where $G$ is an average shear rate for the bulk flow. Later, in order to make the calculation tractable, we shall restrict our analysis to small particles for which $a \ll d$. Finally, for convenience, we shall use convected Cartesian co-ordinates with an origin which is coincident with the centre of the sphere. The equations of motion may thus be expressed in the form

$$
\operatorname{Re}[\partial \mathbf{U} / \partial t+\mathbf{U} \cdot \nabla \mathbf{U}]=\nabla \cdot \mathbf{T}, \quad \nabla \cdot \mathbf{U}=0
$$

where the stress tensor $\mathbf{T}$ for a second-order fluid is

$$
\mathbf{T}=-P \mathbf{I}+\mathbf{A}_{(1)}+\lambda \mathbf{A}_{(1)} \cdot \mathbf{A}_{(1)}+\lambda \epsilon_{1} \mathbf{A}_{(2)}
$$

where

$$
\begin{aligned}
& \mathbf{A}_{(\mathbf{1})}=\nabla \mathbf{U}+\nabla \mathbf{U}^{\mathrm{T}} \\
& \mathbf{A}_{(\mathbf{2})}=\partial \mathbf{A}_{(\mathbf{1})} / \partial t+\mathbf{U} \cdot \nabla \mathbf{A}_{(\mathbf{1})}+\mathbf{A}_{(\mathbf{1})} \cdot \nabla \mathbf{U}^{\mathrm{T}}+\nabla \mathbf{U} \cdot \mathbf{A}_{(\mathbf{1})} .
\end{aligned}
$$

$\mathbf{A}_{(1)}$ is the rate-of-strain tensor and $\mathbf{A}_{(2)}$ is the second Rivlin-Ericksen tensor. The relevant dimensionless parameters are the Reynolds number $R e \equiv \rho_{0} G a^{2} / \mu_{0}$ and the non-Newtonian parameters $\lambda=\phi_{3} G / \mu_{0}$ and $\epsilon_{1}=\phi_{2} / \phi_{3}$, in which $\phi_{2}$ and $\phi_{3}$ are related to the magnitude of the normal stress components in shear flow [see (2.7)]. Available experimental evidence indicates that $\lambda>0$ and $\epsilon_{1}<-0.5$ in most polymeric solutions and melts, these values corresponding to a positive first normal-stress difference and a negative second normal-stress difference in simple shear flow. The case $\epsilon_{1}=-0.5$ corresponds to the so-called Weissenberg fluid, in which the second normal-stress difference is exactly zero.

The dimensionless parameter $\lambda$ is a measure of the intrinsic relaxation time for the suspending fluid relative to the dynamic scale $G^{-1}$. In the present work we consider $\lambda$ to be small so that the constitutive relationship (2.1c) differs only slightly from that of a Newtonian fluid. In addition, we assume that the fluid motion is dynamically slow so that inertial effects may be neglected. More precisely, we require

$$
R e \ll \lambda \ll 1,
$$

so that the Newtonian creeping-motion velocity and pressure fields are modified by nonlinear effects associated with non-zero values of $\lambda$ rather than dynamic inertial effects associated with non-zero values of the Reynolds number. The inequality $R e \ll \lambda$ is satisfied if

$$
\phi_{3} \gg \rho_{0} a^{2}
$$

Thus, for a given fluid, the neglect of dynamic inertia compared with nonNewtonian effects is justified for sufficiently small particles.

The use of a second-order fluid model restricts the present theory to the first, $O(\lambda)$, non-Newtonian contribution to the particle motion. We may, therefore, consider the undisturbed bulk flow to be steady, unidirectional and twodimensional, with a quadratic lateral variation in velocity, even when referred 
to convected co-ordinates fixed at the sphere centre. The equations of motion for the undisturbed flow, subject to these assumptions, are simply

$$
\nabla . \mathbf{I}=\mathbf{0}, \quad \nabla . \mathbf{V}=\mathbf{0},
$$

in which

$$
\begin{aligned}
& \mathbf{\Pi}=-Q \mathbf{I}+\mathbf{E}_{(1)}+\lambda \mathbf{E}_{(1)} \cdot \mathbf{E}_{(1)}+\lambda \epsilon_{1} \mathbf{E}_{(2)} \\
& \mathbf{E}_{(1)}=\nabla \mathbf{V}+\nabla \mathbf{V}^{\mathrm{T}}, \\
& \mathbf{E}_{(2)}=\mathbf{V} \cdot \nabla \mathbf{E}_{(1)}+\mathbf{E}_{(1)} \cdot \nabla \mathbf{V}^{\mathrm{T}}+\nabla \mathbf{V} . \mathbf{E}_{(1)} .
\end{aligned}
$$

A solution to these equations which encompasses both two-dimensional Poiseuille and simple shear flow is

$$
\begin{gathered}
\mathbf{V}=\left(\alpha+\beta z+\gamma z^{2}\right) \mathbf{e}_{x}-\left(\mathbf{U}_{s}\right)_{x} \\
Q=2 \gamma x+4 \gamma\left(\beta z+\gamma z^{2}\right)\left(1+2 \epsilon_{1}\right) \lambda+\text { constant, }
\end{gathered}
$$

where $\left(U_{s}\right)_{x}$ is the velocity of the sphere in the $x$ direction. For a simple shear flow, as depicted in figure $1(\alpha)$, the constants $\alpha, \beta$ and $\gamma$ are

$$
\alpha=V_{w} s, \quad \beta=V_{w} \kappa, \quad \gamma=0,
$$

where $V_{w}$ is velocity of the moving wall, $s$ is the non-dimensionalized distance across the gap from the fixed wall, $d$ is the gap width and $\kappa=a / d$. For a twodimensional Poiseuille flow, as depicted in figure $1(b)$,

$$
\alpha=4 V_{\max } s(1-s), \quad \beta=4 V_{\max }(1-2 s) \kappa, \quad \gamma=-4 V_{\max } \kappa^{2},
$$

where $V_{\max }$ is the maximum velocity at the midpoint between the walls. It may be noted that, for a second-order fluid, the undisturbed velocity field is unchanged from that of a Newtonian fluid having the same viscosity $\mu_{0}$, but does produce a contribution to the isotropic pressure at order $\lambda$. The first and second normalstress differences are given, to $O(\lambda)$, by

$$
\left.\begin{array}{l}
\Pi_{x x}-\Pi_{z z}=-2\left(d V_{x} / d z\right)^{2} \epsilon_{1} \lambda \\
\Pi_{z z}-\Pi_{y y}=\left(d V_{x} / d z\right)^{2}\left(1+2 \epsilon_{1}\right) \lambda
\end{array}\right\}
$$

For the analysis of (2.1), it is convenient to define a disturbance velocity $\mathbf{v}=\mathbf{U}-\mathbf{V}$ and pressure $q=P-Q$. Again, for $O(\lambda)$ calculations, the disturbance motion may be assumed steady, and, in view of (2.2), the governing equations are

$$
\nabla \cdot \pi=0, \quad \nabla \cdot \mathbf{v}=0
$$

in which

$$
\pi=-q \mathbf{l}+\mathbf{e}_{(1)}+\lambda\left[\mathbf{e}_{(1)} \cdot \mathbf{e}_{(1)}+\mathbf{W}_{(1)}\right]+\lambda \epsilon_{1}\left[\mathbf{e}_{(2)}+\mathbf{W}_{(2)}\right]
$$

and

$$
\left.\begin{array}{rl}
\mathbf{e}_{(1)} & =\nabla \mathbf{v}+\nabla \mathbf{v}^{\mathbf{T}}, \\
\mathbf{e}_{(2)} & =\mathbf{v} \cdot \nabla \mathbf{e}_{(1)}+\mathbf{e}_{(1)} \cdot \nabla \mathbf{v}^{\mathrm{T}}+\nabla \mathbf{v} \cdot \mathbf{e}_{(1)}, \\
\mathbf{W}_{(1)} & =\mathbf{E}_{(1)} \cdot \mathbf{e}_{(1)}+\mathbf{e}_{(1)} \cdot \mathbf{E}_{(1)}, \\
\mathbf{W}_{(2)} & =\mathbf{V} \cdot \nabla \mathbf{e}_{(1)}+\mathbf{e}_{(1)} \cdot \nabla \mathbf{V}^{\mathrm{T}}+\nabla \mathbf{V} \cdot \mathbf{e}_{(1)}+\mathbf{v} \cdot \nabla \mathbf{E}_{(1)}+\mathbf{E}_{(1)} \cdot \nabla \mathbf{v}^{\mathbf{T}}+\nabla \mathbf{v} \cdot \mathbf{E}_{(1)} \cdot
\end{array}\right\}
$$

Here $\mathbf{e}_{(1)}$ and $\mathbf{e}_{(2)}$ are respectively the rate-of-strain tensor and the second RivlinEricksen tensor for acceleration of the disturbance flow $\mathbf{v}$, while $\mathbf{W}_{(1)}$ and $\mathbf{W}_{(2)}$ are tensors arising from the interaction of the disturbance flow $\mathbf{v}$ and the bulk 


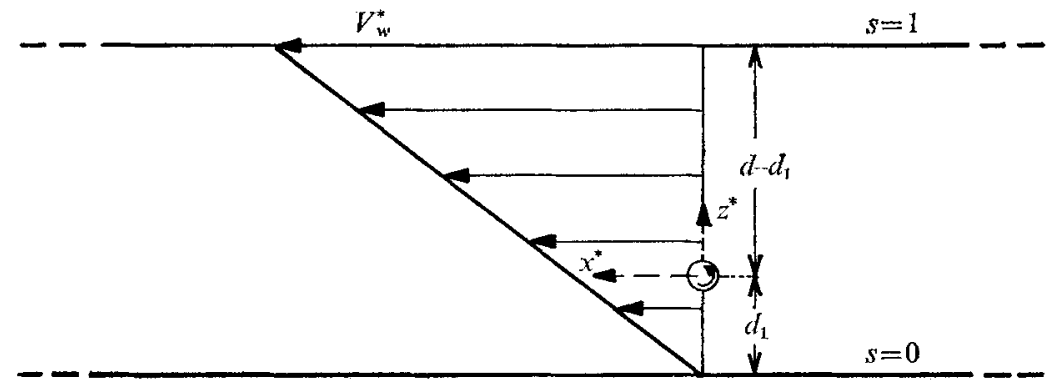

(a)

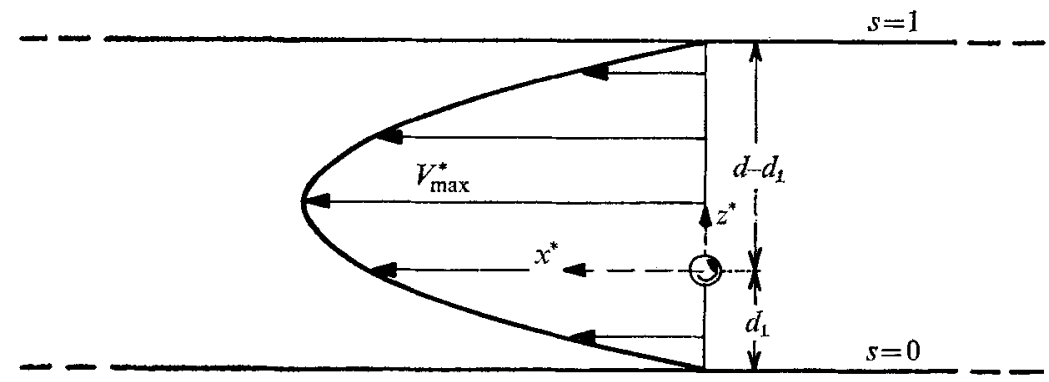

(b)

Frgure 1. The physical system for (a) simple shear flow and (b) two-dimensional Poiseuille flow.

flow V. We seek solutions of (2.8) plus associated boundary conditions, subject to the asymptotic restriction $\lambda \ll 1$. Thus we assume

$$
\begin{aligned}
& \mathbf{v}=\mathbf{v}^{(0)}+\lambda \mathbf{v}^{(1)}+\ldots, \\
& q=q^{(0)}+\lambda q^{(1)}+\ldots .
\end{aligned}
$$

Substituting (2.9) into (2.8) and equating powers of $\lambda$, we obtain governing equations for $v^{(0)}$ and $v^{(1)}$. For $\left(v^{(0)}, q^{(0)}\right)$ we obtain

$$
\nabla \cdot \pi^{(0)}=0, \quad \nabla \cdot \mathbf{v}^{(0)}=0, \quad \pi^{(0)}=-q^{(0)} \mathbf{I}+\mathbf{e}_{(1)}^{(0)},
$$

which is the equation of motion of a Newtonian fluid and can be written in the more familiar form

$$
-\nabla q^{(0)}+\nabla^{2} \mathbf{v}^{(0)}=0, \quad \nabla \cdot \mathbf{v}^{(0)}=0 .
$$

The appropriate boundary conditions are

$$
\begin{aligned}
& \mathbf{v}^{(0)}=\boldsymbol{\Omega}_{s}^{(0)} \times \mathbf{r}-\mathbf{V} \text { on } r=1, \\
& \mathbf{v}^{(0)}=0 \text { on the walls, } \\
& \mathbf{v}^{(0)} \rightarrow 0 \text { as } \mathbf{r} \rightarrow \infty
\end{aligned}
$$


Here $\boldsymbol{\Omega}_{s}^{(0)}$ is the angular velocity of the sphere to $O(1)$. For $\left(\mathbf{v}^{(1)}, q^{(1)}\right)$ we obtain

$$
\begin{gathered}
\nabla \cdot \pi^{(1)}=0, \quad \nabla \cdot \mathbf{v}^{(1)}=0, \\
\boldsymbol{\pi}^{(1)}=-q^{(1)} \mathbf{I}+\mathbf{e}_{(1)}^{(1)}+\boldsymbol{\Sigma}^{(0)}, \\
\boldsymbol{\Sigma}^{(0)}=\mathbf{e}_{(1)}^{(0)} \cdot \mathbf{e}_{(1)}^{(0)}+\mathbf{W}_{(1)}^{(0)}+\epsilon_{1}\left[\mathbf{e}_{(2)}^{(0)}+\mathbf{W}_{(2)}^{(0)}\right],
\end{gathered}
$$

where $\Sigma^{(0)}$ contains only terms from $V$ and $v^{(0)}$. We can also write (2.13) as

$$
-\nabla q^{(1)}+\nabla^{2} \mathbf{v}^{(1)}=-\nabla \cdot \Sigma^{(0)}, \quad \nabla \cdot \mathbf{v}^{(1)}=0 .
$$

The appropriate boundary conditions are

$$
\begin{aligned}
& \mathbf{v}^{(1)}=\boldsymbol{\Omega}_{s}^{(1)} \times \mathbf{r}+\mathbf{U}_{s}^{(1)} \text { on } r=1, \\
& \mathbf{v}^{(1)}=0 \text { on the walls, } \\
& \mathbf{v}^{(1)} \rightarrow 0 \text { as } \mathbf{r} \rightarrow \infty .
\end{aligned}
$$

Here $\boldsymbol{\Omega}_{s}^{(1)}$ and $\mathbf{U}_{s}^{(1)}$ are the angular and translational velocities of the sphere at $O(\lambda)$. All of the variables $\boldsymbol{\Omega}_{\boldsymbol{s}}^{(\mathbf{0})}, \boldsymbol{\Omega}_{\boldsymbol{s}}^{(1)}, \mathbf{U}_{\boldsymbol{s}}^{(\mathbf{0})}$ and $\mathbf{U}_{\boldsymbol{s}}^{(1)}$ are unknown, in general, and must be obtained as part of the solution to the problem. Our present objective is to find the $z$ component of $\mathbf{U}_{s}^{(1)}$, which is the lateral velocity of the sphere induced by the non-Newtonian behaviour of the suspending fluid.

The method employed is analogous to that developed in our earlier evaluation of the inertia-induced lateral velocity in a Newtonian fluid (Ho \& Leal 1974). That is, by using the reciprocal theorem, we show that the lateral velocity $\left(\mathbf{U}_{s}^{(1)}\right)_{z}$ can be calculated without the explicit solution for $\mathbf{v}^{(1)}$. A new velocity field $(\mathbf{u}, p)$ is defined by $\quad \nabla \cdot \mathbf{t}=0, \quad \nabla \cdot \mathbf{u}=0$,

$$
\mathbf{t}=-p \mathbf{I}+\mathbf{a}, \quad \mathbf{a}=\nabla \mathbf{u}+\nabla \mathbf{u}^{\mathrm{T}},
$$

or, in its more familiar form,

$$
\begin{gathered}
-\nabla p+\nabla^{2} \mathbf{u}=0, \quad \nabla \cdot \mathbf{u}=0 \\
\mathbf{u}=\mathbf{e}_{z} \quad \text { on } \quad r=1 \\
\mathbf{u}=0 \quad \text { on the walls, } \\
\mathbf{u} \rightarrow 0 \quad \text { as } \mathbf{r} \rightarrow \infty
\end{gathered}
$$

Combining (2.13a) and (2.16a) and integrating over the entire fluid volume, we obtain

$$
\int_{V_{f}}\left[\mathbf{u} \cdot \nabla \cdot \pi^{(1)}-\mathbf{v}^{(1)} \cdot \nabla \cdot \mathbf{t}\right] d V=0
$$

which can be rearranged to give

$$
\int_{V_{f}} \nabla \cdot\left[\mathbf{u} \cdot \pi^{(1)}-\mathbf{v}^{(1)} \cdot \mathbf{t}\right] d V-\int_{V_{f}}\left[\pi^{(1)}: \nabla \mathbf{u}-\mathbf{t}: \nabla \mathbf{v}^{(1)}\right] d V=0 .
$$

Upon applying the divergence theorem to the first integral and using the definitions of $\pi^{(1)}$ and $t$ in the second integral, (2.19) becomes

$$
\begin{array}{r}
-\int_{A}\left[\mathbf{u} \cdot \pi^{(1)}-\mathbf{v}^{(1)} \cdot \mathbf{t}\right] \cdot \mathbf{n} d A-\int_{V_{f}}\left[\left(-q^{(1)} \mathbf{I}+\mathbf{e}_{(1)}^{(1)}\right): \nabla \mathbf{u}-(-p \mathbf{I}+\mathbf{a}): \nabla \mathbf{v}^{(1)}\right] d V \\
=\int_{V_{f}} \boldsymbol{\Sigma}^{(0)}: \nabla \mathbf{u} d V,
\end{array}
$$


where $\mathbf{n}$ is the outward unit normal to the surfaces. It may easily be shown that the integrand of the second integral is identically zero. Thus application of boundary conditions to the first integral gives the simplified form

$$
\begin{aligned}
-\int_{A}\left(\boldsymbol{\pi}^{(1)} \cdot \mathbf{n}\right) \cdot \mathbf{e}_{z} d A+\mathbf{U}_{s}^{(1)} \cdot \int_{A} \mathbf{t} \cdot \mathbf{n} d A+\int_{A}\left[\mathbf{\Omega}_{s}^{(1)} \times \mathbf{r}\right] \cdot(\mathbf{t} \cdot \mathbf{n}) d A & \\
& =\int_{V_{f}} \boldsymbol{\Sigma}^{(0)}: \nabla \mathbf{u} d V .
\end{aligned}
$$

The first integral is the force on the sphere in the $z$ direction due to $\mathrm{v}^{(1)}$ and is assumed to be zero for a neutrally buoyant sphere since the acceleration of the sphere yields a term of order $\operatorname{Re} \kappa\left(U_{s}\right)_{z}^{2}$. The second integral is the force on the sphere due to $\mathbf{u}$ and is equal to $-6 \pi\left[1+O(\kappa)+O\left(\lambda^{2}\right)\right] \mathbf{e}_{z}$. The third integral is the torque on the sphere due to $\mathbf{u}$ and is identically zero owing to the symmetry of the problem (2.17). Since $\boldsymbol{\Sigma}^{(0)}$ is symmetric, the integrand of the fourth integral can be written as $\boldsymbol{\Sigma}^{0}: \nabla \mathbf{u}=\frac{1}{2} \boldsymbol{\Sigma}^{(0)}: \mathbf{a}$, where $\mathbf{a}=\nabla \mathbf{u}+\nabla \mathbf{u}^{\mathbf{T}}$ is the rate-of-strain tensor for the velocity field $\mathbf{u}$. Therefore (2.21) becomes

$$
6 \pi\left(U_{s}^{(1)}\right)_{z}=-\frac{1}{2} \int_{V_{f}} \boldsymbol{\Sigma}^{(0)}: \mathbf{a} d V
$$

We have shown in the inertial-migration case (Ho \& Leal 1974) that an equivalent result may be obtained for the $O(R e)$ contribution to the lateral migration velocity if one first calculates the force which would be necessary to keep the sphere from migrating, and then calculates the migration velocity by equating this force to the Stokes drag for uniform translation with velocity $\left(U_{s}^{(1)}\right)_{z}$ through a quiescent fluid. Using identical arguments, the equivalence of these two approaches may also be proved in the present case. Thus the force on the sphere in the $z$ direction which is equivalent to $(2.22)$ is simply

$$
F_{L}=-\frac{1}{2} \lambda \int_{V_{J}} \boldsymbol{\Sigma}^{(0)}: \mathbf{a} d V
$$

It may be noted that the integrand in (2.23) has a different form from its counterpart in the inertial-migration problem,

$$
\left.\begin{array}{rl}
F_{L} & =-R e \int_{V_{f}} \mathbf{u} \cdot \mathbf{f} d V, \\
\mathbf{f} & =\mathbf{v}^{(0)} \cdot \nabla \mathbf{V}+\mathbf{V} \cdot \nabla \mathbf{v}^{(0)}+\mathbf{v}^{(0)} \cdot \nabla \mathbf{v}^{(0)}
\end{array}\right\}
$$

and from the more cumbersome force expression which was used by Leal (1975, equation 29) in a recent calculation of the motion of slender rod-like particles in a second-order fluid. In particular, (2.24) has the inhomogeneous term $f$ of the $O(R e)$ equations of motion dotted directly with $\mathbf{u}$, whereas the present form (2.23) involves the double dot product of the inhomogeneous part of the stress tensor at $O(\lambda)$ [see (2.13)] with the rate-of-strain tensor corresponding to $\mathbf{u}$. Leal (1975) has shown that, if a were replaced in (2.23) by $\mathbf{u}$ and $\boldsymbol{\Sigma}^{(0)}$ by $\nabla . \boldsymbol{\Sigma}^{(0)}$ in analogy with (2.24), an additional integral of $\mathbf{e}_{(1)}^{(0)}$. $\mathbf{e}_{(1)}^{(0)}+\epsilon_{1} \mathbf{e}_{(2)}^{(0)}$ over the sphere surface would be required to obtain $F_{L}$. The difference between the forms (2.23) 
and (2.24) is thus introduced primarily as a matter of computational convenience. The forms (2.23) and (2.24) may also be seen to arise as equivalent natural choices if we consider the overall rate of work done on a fluid volume. In dimensional terms, this quantity can be expressed as the sum of two terms (see Batchelor 1967, p. 152):

$$
\int_{V_{f}}\left[\rho \mathbf{v} \cdot \mathbf{g}+\frac{1}{2} \pi: \mathbf{e}_{(1)}\right] d V
$$

where $\mathbf{g}=\partial \mathbf{v} / \partial t+\mathbf{v} \cdot \nabla \mathbf{v}$ and all other quantities have their usual meanings. If the rate of work is non-dimensionalized by $\mu V^{2} L$ ( $V$ and $L$ are the characteristic velocity and length respectively), the first part of the integral (2.25) becomes (in dimensionless form)

$$
R e \int_{V_{f}} \mathbf{v} \cdot \mathbf{g} d V
$$

which is similar to (2.24). For a second-order fluid (in which the dimensionless stress is given by $\left.\pi=\mathbf{e}_{(1)}+\lambda \sigma\right)$, the second part of the integral (2.25) has a nonNewtonian contribution given by (in dimensionless form)

which is similar to $(2.23)$.

$$
\frac{1}{2} \lambda \int_{V_{f}} \boldsymbol{\sigma}: \mathbf{e}_{(1)} d V
$$

\section{Evaluation of the lateral force}

In order to evaluate the lateral force $F_{L}$ using (2.23), the product $\Sigma^{(0)}:$ a must be integrated over the entire volume of fluid which is outside the sphere and bounded by the two walls. Thus, in general, solutions of (2.11), (2.12) and (2.17) for $\mathbf{v}^{(0)}$ and $\mathbf{u}$ are required throughout the entire fluid domain. Although the derivation of these solutions would be extremely difficult in the general case, approximate analytical results can be obtained for small particles, i.e. $\kappa \equiv a / d \ll 1$, via the well-known method of reflexions. Thus we shall limit our subsequent analysis to $\kappa \ll 1$, in addition to the condition $(2.2 a)$ which was adopted earlier. The solutions for $\mathbf{v}^{(0)}$ and $\mathbf{u}$ were derived in our earlier analysis of the inertial-migration problem (Ho \& Leal 1974) and, in view of their length, we shall not repeat them here, but rather concentrate our attention on the evaluation of the volume integral in (2.23).

It is convenient, as in the case of inertial migration, to divide the volume of integration $V_{f}$ into two parts $V_{1}$ and $V_{2}$, where

$$
\left.\begin{array}{l}
V_{1}=\left\{\mathbf{r} \mid 1 \leqslant r<\lambda \kappa^{x-1}\right\}, \\
V_{2}=\left\{\mathbf{r} \mid \lambda \kappa^{\chi-1} \leqslant r<\infty,-s \kappa^{-1} \leqslant z \leqslant(1-s) \kappa^{-1}\right\} .
\end{array}\right\}
$$

Here $\lambda$ is a constant of order $\kappa^{0}$ and $0<\chi<1$. Hence

$$
F_{L}=-\frac{1}{2} \lambda\left\{\int_{V_{2}} \boldsymbol{\Sigma}^{(0)}: \mathbf{a} d V+\int_{V_{\mathbf{3}}} \boldsymbol{\Sigma}^{(0)}: \mathbf{a} d V\right\}
$$

In contrast to the previous case (Ho \& Leal 1974), the dominant contribution to (3.2) will be shown to arise from the integration over $V_{1}$, i.e. from the region 
near the sphere. In order to demonstrate this fact we consider the order of magnitude of each of the two integrals in (3.2).

Let us first examine the integral over $V_{1}$, i.e. $F_{L 1}$. In this region, it can be shown by using the solutions for $v^{(0)}$ and $\mathbf{u}$ from Ho \& Leal (1974) that the integrand behaves like

$$
\begin{aligned}
\mathbf{\Sigma}^{(0)}: \mathbf{a}=\beta^{2}\left[X_{1}\right. & \left.\left(\frac{z}{r^{6}}, \ldots\right)+\epsilon_{1} X_{2}\left(\frac{z}{r^{6}}, \ldots\right)\right] \\
& +\beta \gamma\left[Y_{1}\left(\frac{1}{r^{4}}, \frac{1}{r^{6}}, \frac{1}{r^{8}}, \ldots\right)+\epsilon_{1} Y_{2}\left(\frac{1}{r^{4}}, \frac{1}{r^{6}}, \frac{1}{r^{8}}, \ldots\right)+O\left(\kappa^{4}\right) .\right.
\end{aligned}
$$

The functions $Y_{1}$ and $Y_{2}$ depend on the undisturbed flow $\mathrm{V}$ and on $\mathbf{v}^{(0)}$ and $\mathbf{u}$ for the motion of a sphere in an unbounded fluid domain. That is, the reflexion of this solution at the walls and the resulting higher-order corrections at the walls and at the sphere surface contribute only to $X_{1}$ and $X_{2}$ and the $O\left(\kappa^{4}\right)$ terms. Since $\beta^{2} \sim \kappa^{2}$ and $\beta \gamma \sim \kappa^{3}$, the $O\left(\kappa^{4}\right)$ terms may be neglected for $\kappa \ll 1$. Furthermore, the integral of $X_{1}$ and $X_{2}$ over the spherical shell $1 \leqslant r<\lambda \kappa^{x-1}$ is identically zero. Thus

$$
\begin{aligned}
F_{L 1} & =\int_{r=1}^{r \leqslant \lambda \kappa^{x-1}} \Sigma^{(0)}: \mathbf{a} d V \\
& =\int_{r=1}^{r \leqslant \lambda \kappa} x^{x-1} \beta \gamma\left[Y_{1}\left(\frac{1}{r^{4}}, \frac{1}{r^{6}}, \frac{1}{r^{8}}, \ldots\right)+\epsilon_{1} Y_{2}\left(\frac{1}{r^{4}}, \frac{1}{r^{6}}, \frac{1}{r^{8}}, \ldots\right)\right] d V+O\left(\kappa^{4}\right) .
\end{aligned}
$$

Finally, denoting the indefinite integral of $Y_{1}+\epsilon_{1} Y_{2}$ as $\Psi^{\circ}\left(r^{-1}, r^{-3}, r^{-5}, \ldots ; \epsilon_{1}\right)$ and noting that the upper limit of (3.4) can be replaced by $\infty$ with an error $o\left(\kappa^{3}\right)$, we obtain

$$
\begin{aligned}
F_{L 1} & =\left.\beta \gamma \Psi\left(\frac{1}{r}, \frac{1}{r^{3}}, \frac{1}{r^{5}}, \ldots ; \epsilon_{1}\right)\right|_{r=1} ^{r=\infty}+o\left(\kappa^{3}\right) \\
& =\beta \gamma\left(\Psi_{1}+\epsilon_{1} \Psi_{2}\right)+o\left(\kappa^{3}\right) .
\end{aligned}
$$

Let us now turn to $F_{L 2}$. In the region $V_{2}$, it is appropriate to use the 'outer' variable $r^{\prime}=\kappa r$. Thus, transforming the integral expression for $F_{L 2}$ into this outer co-ordinate system, we obtain

$$
F_{L 2}=\kappa^{-3} \int_{r^{\prime}=\lambda \kappa^{x}}^{r^{\prime}=O(1)} \Sigma^{(0)^{\prime}}: \mathbf{a}^{\prime} d V^{\prime}
$$

where a prime signifies the use of outer variables. Again using the solutions of Ho \& Leal (1974) it may be shown that $\boldsymbol{\Sigma}^{(0)^{\prime}}: \mathbf{a}^{\prime}=O\left(\kappa^{7}\right)$. It thus follows from (3.6) that $F_{L 2}=o\left(\kappa^{3}\right)$.

We thus see that the dominant $O\left(\kappa^{3}\right)$ contribution to the lateral force is due entirely to $F_{L 1}$, which is itself dominated, for small $\kappa$, by the disturbance velocity fields for the sphere in an infinite fluid domain, i.e.

$$
F_{L} \sim-\frac{1}{2} \lambda \beta \gamma\left(\Psi_{1}+\epsilon_{1} \Psi_{2}\right)+o\left(\kappa^{3}\right) .
$$

Unlike the inertial case, the reflexion of the infinite-domain solution off the walls yields only higher-order corrections to $F_{L}$. Thus, in calculating the lateral force to $O\left(\kappa^{3}\right)$ for a sphere not too close to a wall, the only role played by the walls is 
in the establishment of the undisturbed profile $\mathbf{V}$. In addition, it should be noted from (3.7) that the lateral force $F_{L}$ is proportional to $\beta \gamma$. Hence in the case of simple shear $(\gamma=0)$ no lateral migration" will occur to $O\left(\kappa^{3}\right)$.

In order to obtain more quantitative results, the coefficients $\Psi_{1_{2}}^{P}$ and $\Psi_{2}$ must be evaluated. From the definition of $\Sigma_{i j}^{(0)}$, these are simply

$$
\begin{aligned}
& \Psi_{1}=\frac{1}{\beta \gamma} \int_{r=1}^{\infty}\left[\mathbf{w}_{(1)}^{(0)}+\mathbf{e}_{(1)}^{(0)} \cdot \mathbf{e}_{(1)}^{(0)}\right]: \nabla \mathbf{u} d V, \\
& \Psi_{2}=\frac{1}{\beta \gamma} \int_{r=1}^{\infty}\left[\mathbf{w}_{(2)}^{(0)}+\mathbf{e}_{(2)}^{(0)}\right]: \nabla \mathbf{u} d V .
\end{aligned}
$$

Straightforward, though tedious, evaluation of these terms gives

$$
\Psi_{1}=\left(-\frac{20}{3}+0\right) \pi, \quad \Psi_{2}=(-20+0) \pi,
$$

and the lateral force is

$$
F_{L}=10 \pi \beta \gamma \lambda\left\{\frac{1}{3}+\epsilon_{1}\right\}=-\pi \beta \gamma \lambda N\left(\epsilon_{1}\right)+o\left(\kappa^{3}\right) .
$$

In $(3.8 a)$, the first term is the contribution from $w_{(1)}^{(0)}$. The contribution from $\mathbf{e}_{(1)}^{(0)} \cdot \mathbf{e}_{(1)}^{(0)}$ is identically zero. Likewise, in $(3.8 b)$, the first term is the contribution from $\mathbf{w}_{(2)}^{(0)}$, while the second, corresponding to $\mathbf{e}_{(2)}^{(0)}$, is zero. It is of interest to note that the contributions of the extra, non-Newtonian stress $\boldsymbol{\Sigma}^{(0)}$ to $F_{L}$ are completely associated with the interaction of the bulk flow and the disturbance flow. Also, we may recall that the lateral force for a neutrally buoyant sphere with no external torque in the inertial case stems from the stresslet contribution to the far-field behaviour of the disturbance flow. Here, in the non-Newtonian case, the lateral force depends on the disturbance velocity field close to the sphere, and all of the velocity terms coming from $D_{1}, E_{1}, F_{1}, G_{1}, H_{1}$ and $B_{1}$ in the disturbance velocity $v^{(0)}$ (see Ho \& Leal 1974, §3) contribute to the same order of magnitude. The contributions from the Stokeslet term $A_{1}$ and the couplet term $C_{1}$ are asymptotically small and thus neglected for a neutrally buoyant, freely rotating particle. However, closer examination of these terms is useful since it leads to criteria for the neglect of external body forces and couples. In order to neglect the contribution from $A_{1}$, it can be shown from $v^{(0)}$ that we require

$$
\gamma \gg A_{1}
$$

For a neutrally buoyant sphere $A_{1} \sim \kappa^{3}$ while $\gamma \sim \kappa^{2}$. However, with an external body force $F_{x}$ acting on the sphere in the $x$ direction, $A_{1} \sim\left|F_{x}\right|$, and thus the contribution from $A_{1}$ can be neglected only if

$$
\gamma \gg\left|F_{x}\right|
$$

If the body force is gravitational, then in dimensional quantities $(3.10 b)$ becomes

$$
\gamma^{*} \gg\left|\rho_{s}-\rho_{0}\right| g \mid \mu_{0}
$$

which for the case of plane Poiseuille flow is

$$
V_{\max }^{*} \gg d^{2}\left|\rho_{s}-\rho_{0}\right| g / \mu_{0} \text {. }
$$

It is coincidental that the same criteria for neglect of body-force contributions 
was obtained for the inertial-migration case (see equation (5.23)) of Ho \& Leal 1974). It can also be shown that, in order to neglect the couplet term $C_{1}$, we require

$$
\beta \gg C_{1} \text {. }
$$

For a freely rotating sphere $C_{1} \sim \kappa^{4}$, and the condition is satisfied. On the other hand, for a non-rotating sphere with $\Omega_{s y}=0, C_{1}$ is $\frac{1}{2} \beta$ and hence contributes to $F_{L}$ at the same order of magnitude as the rest of the terms $D_{1}, E_{1}, F_{1}, \ldots$. Again, this is true in the inertial-migration case. It thus also follows that the present result for migration of a single particle is applicable for a suspension of spheres provided conditions (7.2) and (7.3) of Ho \& Leal (1974) are satisfied.

Finally, it may be noted that the approximate condition $(2.2 a)$ for neglect of the inertial contribution to the lateral force compared with the non-Newtonian contribution may now be improved by direct comparison of the magnitudes of the predicted lateral force in each case. In the present theory, we have shown that the lateral force due to the non-Newtonian effect is of order $\lambda \kappa^{3}$, while the analysis of Ho \& Leal (1974) produced an inertial contribution of order Re ${ }^{2}$. Thus a more accurate form of the condition $(2.2 a)$ is

or in dimensional terms

$$
\lambda \kappa \gg R e,
$$

$$
\phi_{3} \gg \rho_{0} a d \text {. }
$$

The expression (3.9) for $F_{L}$ is the main result of the present analysis, and is valid whenever conditions (3.11)-(3.13) are satisfied. It shows that the direction and magnitude of the lateral migration depend on the magnitude and sign of the normal-stress parameters $\lambda$ and $\epsilon_{1}$. The majority of available viscometric and theoretical studies (summarized in Leal 1975) support the conclusion that the first normal-stress difference in simple shear flow is positive, while the second normal-stress difference is negative and approximately $10-20 \%$ of the first normal-stress difference in magnitude. Thus, referring to (2.7), it may be seen that $\lambda>0$ and $\epsilon_{1}<-0.5$. It follows from (3.9) that $F_{L}$ is strictly negative, so that particle migration is always in the direction of least (absolute) shear rate. Thus the equilibrium position of particles in Poiseuille flow is midway between the walls and in Couette flow is at the outer cylinder (irrespective of which cylinder is rotating or the direction of relative rotation if both cylinders rotate).

Finally, an alternative, but completely equivalent, expression for $F_{L}$ may be obtained which separates the contributions of the first and second normal-stress differences $N_{1}=-2 \epsilon_{1} \lambda$ and $N_{2}=\left(1+2 \epsilon_{1}\right) \lambda$ [cf. (2.7)]:

$$
F_{L}=-\frac{5}{3} \pi \beta \gamma\left\{N_{1}-2 N_{2}\right\}
$$

The two terms in the brackets have the same sign since $N_{1}>0$ and $N_{2}<0$, meaning that the two normal-stress contributions are acting in the same direction. However, the dominant effect is that associated with the first normal-stress difference.

Qualitatively, the dominant first normal-stress difference represents a tension in the streamline direction which increases with the shear rate, being proportional to the square of the shear rate in the second-order fluid approximation. Previous 
workers have suggested that lateral gradients of the normal stresses in the undisturbed flow are responsible for the observed migration. As we have noted earlier, this explanation cannot be directly applicable since the presence of the sphere causes a strong local disturbance flow as the fluid passes around it. The concept of tensioned streamlines is not altogether lost, however, because the composite disturbed flow exhibits no classical stagnation point for the freely rotating sphere, and is thus locally still primarily a shear flow. $\dagger$ Furthermore, it may be expected that the disturbed flow will exhibit a gradient of mean shear rate across the sphere in the same sense as the undisturbed flow. On each side of the sphere, the bowed streamlines under tension exert a sideways (lateral) 'hoop' thrust. A plausible explanation for the theoretical result is that the 'hoop' thrust mechanism is responsible for the particle motion, with the net force coming from the side of the sphere with highest shear rates. Unfortunately, without a detailed description of the disturbance velocity and stress fields for a second-order fluid, a conclusive test of this suggestion is not possible. However, if it is assumed to be correct (as we believe it to be), then a spherical particle subjected to unidirectional shear flow of a second-order fluid would always be expected to migrate laterally in the direction of decreasing absolute shear rate, regardless of the detailed form of the undisturbed velocity profile.

Although none of the available experimental studies was carried out in a regime for which the second-order fluid model is strictly applicable, our present theoretical results are at least in qualitative agreement with the observations of Karnis \& Mason (1966), which were made in strongly viscoelastic solutions. Since the only relevant non-Newtonian characteristic of the second-order fluid is the existence of non-zero normal stress components, it may perhaps be inferred that the migration phenomenon in a fully viscoelastic fluid which exhibits both a shear-thinning viscosity and non-zero normal stresses is dominated by the normal-stress contributions. A stronger statement would require a quantitative comparison of particle trajectories with measurements in a fully characterized fluid. In the following section, we provide the necessary theoretical results for the trajectories, and show that they agree qualitatively with the available trajectory measurements of Karnis \& Mason (1966). Unfortunately, however, the desired quantitative comparison could not be made with any certainty because of a lack of quantitative rheological data for the test fluid.

\section{Particle trajectories}

In order to facilitate a more quantitative comparison between the present theory and experiments, it is necessary to calculate the particle trajectories. To achieve this, we use the (dimensional) equation for the lateral velocity,

$$
\left(U_{s}\right)_{z}^{*}=d\left(d s / d t^{*}\right)=-\phi_{3} \beta^{*} \gamma^{*} a^{2} N\left(\epsilon_{1}\right) / 6 \mu_{0},
$$

and, for the axial velocity,

$$
\left(U_{s}\right)_{z}^{*}=d\left(d x^{\prime} / d t^{*}\right)=\alpha^{*},
$$

$\dagger$ We are indebted to one of the referees for this remark. 


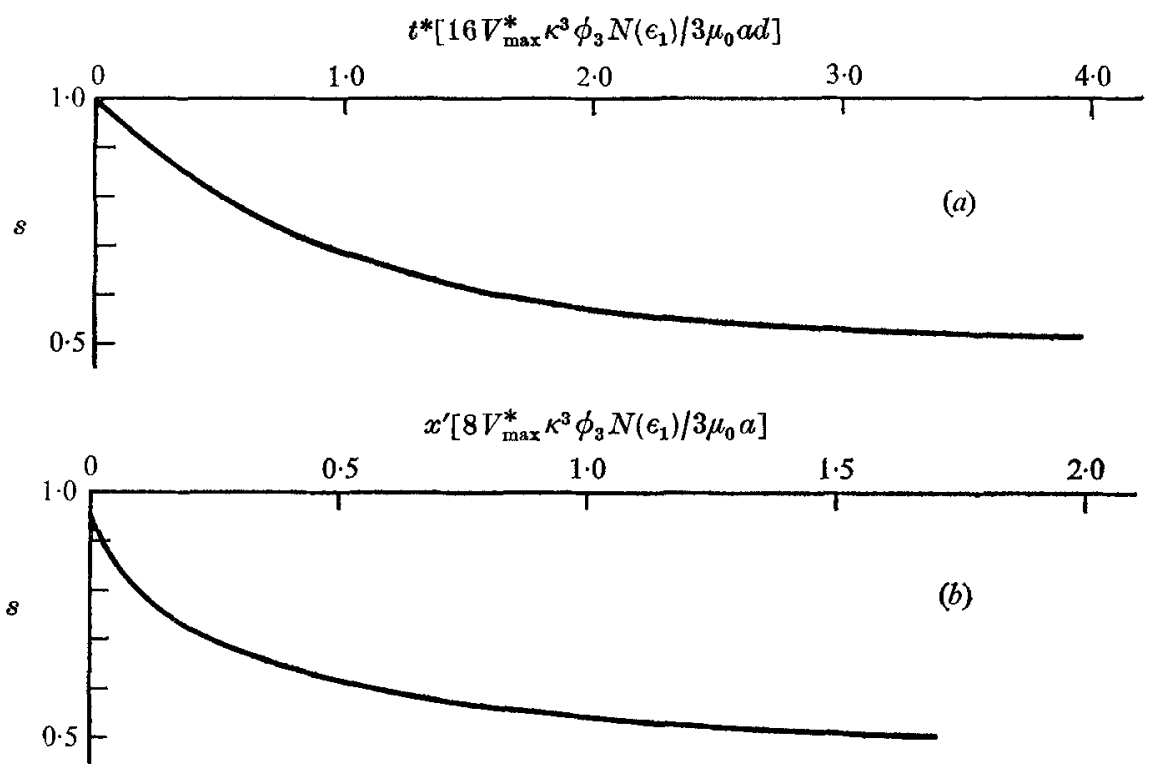

Fradre 2. Particle trajectory for two-dimensional Poiseuille flow: lateral position vs. (a) time and (b) axial position.

to obtain the differential trajectory equations

$$
d t^{*}=-\frac{6 d \mu_{0} d s}{\phi_{3} \beta^{*} \gamma^{*} a^{2} N\left(\epsilon_{1}\right)}, \quad d x^{\prime}=-\frac{6 \alpha^{*} \mu_{0} d s}{\phi_{3} \beta^{*} \gamma^{*} a^{2} N\left(\epsilon_{1}\right)} .
$$

Here $x^{\prime}=\kappa x, t^{*}$ is the dimensional time, $\alpha^{*}, \beta^{*}$ and $\gamma^{*}$ are the dimensional forms of $\alpha, \beta$ and $\gamma$, respectively. Upon integration, $(4.3 a, b)$ become

$$
t^{*}-t_{0}^{*}=-\frac{6 \mu_{0} d}{\phi_{3} a^{2} N\left(\epsilon_{1}\right)} \int_{s_{0}}^{s} \frac{d s^{\prime}}{\beta^{*}\left(s^{\prime}\right) \gamma^{*}\left(s^{\prime}\right)}
$$

and

$$
x^{\prime}-x_{0}^{\prime}=-\frac{6 \mu_{0}}{\phi_{3} a^{2} N\left(\epsilon_{1}\right)} \int_{s_{0}}^{s} \frac{\alpha^{*}\left(s^{\prime}\right) d s^{\prime}}{\beta^{*}\left(s^{\prime}\right) \gamma^{*}\left(s^{\prime}\right)} \text {. }
$$

Equation (4.4a) gives the lateral position $s$ as a function of time, while (4.4b) gives the lateral position as a function of axial position in the flow domain. Let us first consider the case of plane Poiseuille flow, for which

$$
\alpha^{*}=4 V_{\max }^{*} s(1-s), \quad \beta^{*}=4 V_{\max }^{*}(1-2 s) / d, \quad \gamma^{*}=-4 V_{\max }^{*} / d^{2} .
$$

In this case, $(4.4 a, b)$ give

$$
\begin{gathered}
-\ln \left(\frac{1-2 s}{1-2 s_{0}}\right)=\frac{16 V_{\max }^{* 2} \kappa^{3} \phi_{3} N\left(\epsilon_{1}\right)}{3 \mu_{0} a d}\left(t^{*}-t_{0}^{*}\right) \\
\left(s^{2}-s_{0}^{2}\right)-\left(s-s_{0}\right)-\frac{1}{2} \ln \left(\frac{1-2 s}{1-2 s_{0}}\right)=\frac{8 V_{\max }^{*} \kappa^{3} \phi_{3} N\left(\epsilon_{1}\right)}{3 \mu_{0} a}\left(x^{\prime}-x_{0}^{\prime}\right)
\end{gathered}
$$

These trajectory equations are plotted in figures $2(a)$ and $(b)$. So far as we are aware, no experiments have been reported on migration in two-dimensional Poiseuille flow of a non-Newtonian fluid. However, the predicted results do agree 
qualitatively with the trajectories measured in three-dimensional Poiseuille flow by Karnis \& Mason (1966), as may be seen by comparing their figure 4(a) with our figure $2(b)$. Although quantitative agreement would not be expected, it is encouraging that substitution of the experimental parameters into (4.6b) under the assumption that $\epsilon_{1}=-0.55$ does yield reasonable agreement with the experiments for the order of magnitude of the axial distance travelled between an initial lateral position $s_{0}=0.24$ and a final position $s_{f}=0 \cdot 43$. It should, however, be emphasized that no measurements were made of the second normalstress coefficient (requiring us to assume $\epsilon_{1}=0.55$; see the estimate of $\epsilon_{1}$ in $\S 3$ ), that the other rheological data were not measured by Karnis \& Mason (1966), but rather adapted from Brodnyan, Gaskins \& Philippoff (1957), and that the fluid used in the experiments was sufficiently non-Newtonian that the bulk velocity profile differed substantially from the assumed parabolic shape. For these reasons, we have not attempted any more detailed comparison, nor have we formulated the theory for the three-dimensional case in spite of the fact that the analysis is an obvious and straightforward extension of the present work.

The only other experimental observations which are relevant to the present theory are for Couette flow, also reported by Karnis \& Mason (1966). The present theory shows that in simple shear flow, where $\gamma=0$, there is no lateral migration to $O\left(\kappa^{3}\right)$. However, in Couette flow, the small curvature of the velocity profile causes $\gamma$ to be non-zero and thus yields lateral migration. It can be shown that in all cases, whether one or both cylinders are rotating in either the same or the opposite sense, the shear rate always has a minimum absolute value at the outer cylinder wall, which is thus the expected destination of migration according to the present theory. However, owing to detailed differences in the bulk velocity profiles for different combinations of cylinder rotation, the magnitude of the migration velocity will differ from case to case. Both of these features (i.e. outward migration and a dependence of the magnitude on the sense and magnitude of rotation of the individual cylinders) were observed by Karnis \& Mason (1966), who studied the two simplest cases in which (1) the inner cylinder was rotated holding the outer stationary and (2) the outer cylinder was rotated holding the inner stationary. For simplicity, we shall confine our present discussion to these two limiting cases, which we shall refer to as case 1 and case 2, respectively. We denote the radii of the inner and outer cylinders as $r_{1}$ and $r_{2}$, and the gap width as $d=r_{2}-r_{1}$. A dimensionless measure of the degree of profile curvature is

$$
R_{1}=r_{2}\left(r_{2}-r_{1}\right) / r_{1}\left(r_{1}+r_{2}\right) \text { for case } 1
$$

and

$$
R_{2}=r_{1}\left(r_{2}-r_{1}\right) / r_{2}\left(r_{1}+r_{2}\right) \text { for case } 2 \text {. }
$$

Provided $R_{1} \ll 1$ and $R_{2} \ll 1$, we can approximate the (dimensional) undisturbed velocity profile in the general form [from (2.4)]

$$
V^{*}\left(z^{*}\right)=\alpha^{*}+\beta^{*} z^{*}+\gamma^{*} z^{* 2},
$$

in which $z^{*}$ is the axis perpendicular to the walls and directed towards the rotating cylinder. For case 1 , the outer, stationary cylinder is thus represented by $s=0$ and the inner, rotating cylinder by $s=1$. For case 2 , the outer cylinder 
Case 1

$$
\begin{array}{ll}
\alpha^{*} & V_{w}^{*} s\left[1-R_{1}(1-s)\right] \\
\beta^{*} & V_{w}^{*}\left[1-R_{1}(1-2 s)\right] / d \\
\gamma^{*} & V_{w}^{*} R_{1} / d^{2}
\end{array}
$$

Case 2

$$
\begin{aligned}
& V_{w}^{*} s\left[1+R_{2}(1-s)\right] \\
& V_{w}^{*}\left[1+R_{2}(1-2 s)\right] / d \\
& -V_{w}^{*} R_{2} / d^{2}
\end{aligned}
$$

TABIE 1. The coefficients $\alpha^{*}, \beta^{*}$ and $\gamma^{*}$ for Couette flow. Case 1, outer cylinder stationary and inner cylinder rotating; case 2 , outer cylinder rotating and inner cylinder stationary

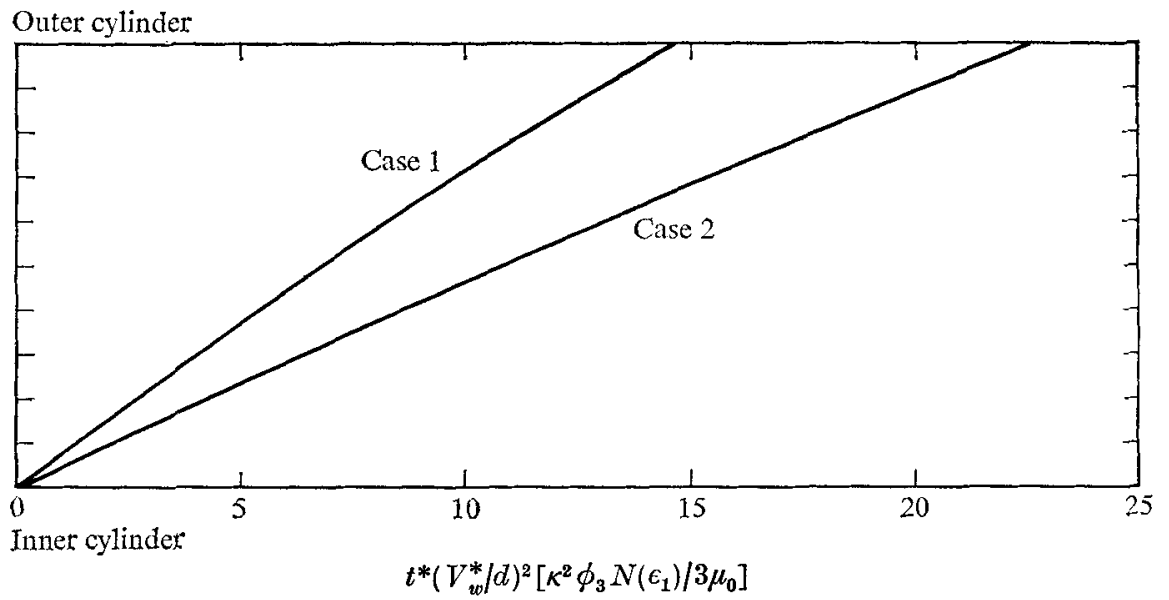

Figure 3. Particle trajectory for Conette flow with $R_{1}=0.138$ and $R_{2}=0.0884$. Case 1 , outer cylinder stationary and inner cylinder rotating; case 2, outer cylinder rotating and inner cylinder stationary.

is $s=1$ and the inner cylinder $s=0$. The coefficients $\alpha^{*}, \beta^{*}$ and $\gamma^{*}$ in each case are given in table 1 . The approximation inherent in (4.7) takes into account the changing shear rate across the gap but neglects the curvature of the walls. Given (4.7), the migration velocities and time trajectories are easily obtained by substituting $\beta^{*}$ and $\gamma^{*}$ into the general expressions $(4.1)$ and $(4.4 a)$. The results are

$$
\begin{gathered}
\left(U_{s}\right)_{z}^{*}=-\frac{\kappa^{3} V_{w}^{* 2} \phi_{3} N\left(\epsilon_{1}\right)}{6 \mu_{0} a} R_{1}\left[1-R_{1}(1-2 s)\right], \\
\frac{1}{3}\left(\frac{V_{w}^{*}}{d}\right)^{2} \frac{\kappa^{2} \phi_{3} N\left(\epsilon_{1}\right)}{\mu_{0}}\left(t^{*}-t_{0}^{*}\right)=-\frac{1}{R_{1}^{2}} \ln \left[\frac{1-R_{1}(1-2 s)}{1-R_{1}\left(1-2 s_{0}\right)}\right]
\end{gathered}
$$

for case 1 and

$$
\begin{gathered}
\left(U_{s}\right)_{z}^{*}=+\frac{\kappa^{3} V_{w}^{* 2} \phi_{3} N\left(\epsilon_{1}\right)}{6 \mu_{0} a} R_{2}\left[1+R_{2}(1-2 s)\right], \\
\frac{1}{3}\left(\frac{V_{w}^{*}}{d}\right)^{2} \frac{\kappa^{2} \phi_{3} N\left(\epsilon_{1}\right)}{\mu_{0}}\left(t^{*}-t_{0}^{*}\right)=-\frac{1}{R_{2}^{2}} \ln \left[\frac{1+R_{2}(1-2 s)}{1+R_{2}\left(1-2 s_{0}\right)}\right]
\end{gathered}
$$

for case 2 . The trajectories $(4.8 b)$ and $(4.9 b)$ are plotted in figure 3 for $R_{1}=0.138$ and $R_{2}=0.0884$, the values used in Karnis \& Mason's experiments. Again, qualitative agreement is found between the theory and experiment for $\kappa=0.012$, the smallest value used by Karnis \& Mason. However, the rate of migration 
which was measured seems to increase too slowly with $\kappa$ when compared with the present theoretical result. One possible explanation for this is that the larger value used, $\kappa=0.056$, is simply too large for the present theory to be applicable. This speculation is, in fact, supported by the trajectory data (figure 8 of Karnis \& Mason 1966), which show a definite wall effect over almost the whole span in this case. It may also be noted that the experimental values of $R_{1}$ and $R_{2}$ are fairly large for the approximate linearization of the flow geometry to be accurate. Finally, the same difficulties with regard to the existence of reliable rheological data are present here as were previously noted in the Poiseuille flow case.

The authors are particularly grateful to $P$. Chan, who found an algebraic error in an earlier draft of this paper. The work reported here is supported by National Science Foundation Grants GK35468 and Eng 74-17590.

\section{REFERENCES}

Bartram, E. 1973 M.Sc. thesis, MoGill University.

Batchelor, G. K. 1967 An Introduction to Fluid Dynamics. Cambridge University Press. Brodnyan, J. G., Gaskins, F. H. \& Phulproff, W. 1957 Trans. Soc. Rheol. 1, 109.

GaUthier, F., Goldsmith, H. L. \& Mason, S. G. 1971 a Rheol. Acta, 10, 344.

GaUthier, F., Goldsmith, H. L. \& Mason, S. G. $1971 b$ Trans. Soc. Rheol. 15, 297.

Highgate, D. J. \& WhoRlow, R. W. 1968 In Polymer Systems: Deformation and Flow (ed. R. E. Wetton \& R. W. Whorlow), p. 251. Macmillan.

Hrghgate, D. J. \& Whorlow, R. W. 1969 Rheol. Acta, 8, 142.

Ho, B. P. \& LeaL, L. G. 1974 J. Fluid Mech. 65, 365.

KarNis, A. \& Mason, S. G. 1966 Trans. Soc. Rheol. 10, 571.

LEAL, L. G. 1975 J. Flwid Mech. 69, 305.

Segré, G. \& Stlberbeirg, A. 1963 J. Colloid Sci. 18, 312. 